\title{
Die Spontaneität des Verstandes bei Kant und einigen Neokantianern
}

\section{Citation}

Boyle, Matthew. 2015. “Die Spontaneität Des Verstandes Bei Kant Und Einigen Neokantianern." Deutsche Zeitschrift Für Philosophie 63 (4) (January 1). doi:10.1515/dzph-2015-0050.

\section{Published Version}

doi:10.1515/dzph-2015-0050

\section{Permanent link}

http://nrs.harvard.edu/urn-3:HUL.InstRepos:33723555

\section{Terms of Use}

This article was downloaded from Harvard University's DASH repository, and is made available under the terms and conditions applicable to Open Access Policy Articles, as set forth at http:// nrs.harvard.edu/urn-3:HUL.InstRepos:dash.current.terms-of-use\#OAP

\section{Share Your Story}

The Harvard community has made this article openly available.

Please share how this access benefits you. Submit a story.

\section{Accessibility}


Matthew Boyle

Emerson Hall 313

Department of Philosophy

Harvard University

Cambridge MA02138boyle2@fas.harvard.edu

Keywords: Kant, spontaneity, belief, cognitive agency

\section{Die Spontaneität des Verstandes bei Kant und einigen Neokantianern}

„Wollen wir die Rezeptivität unseres Gemüts, Vorstellungen zu empfangen, so fern es auf irgend eine Weise affiziert wird, Sinnlichkeit nennen: so ist dagegen das Vermögen, Vorstellungen selbst hervorzubringen, oder die Spontaneität des Erkenntnisses, der Verstand.“1

\section{Einleitung}

Kant charakterisiert unseren menschlichen Verstand - das Vermögen, das uns in die Lage versetzt, Begriffe zu bilden und diese in Urteilen anzuwenden - bekanntermaßen als ein ,spontanes“ Vermögen. Während die „Sinnlichkeit“ das Vermögen ist, das Vorstellungen „empfängt“, indem es von Gegenständen affiziert wird, gilt der Verstand als das Vermögen, durch das unser Geist Vorstellungen selbst „hervorbringt“. Was heißt es aber für ein kognitives Vermögen, „Vorstellungen selbst hervorzubringen“? Und wie passt das, was auch immer hiermit gemeint ist, mit der für Kants Denken ebenso zentralen Idee zusammen, dass unser Verstand kein Vermögen ist, sich Dinge bloß auszudenken, sondern das Vermögen, sinnlich gegebene Gegenstände zu erfassen? Wo ist Raum für Freiheit, geschweige denn Selbsttätigkeit, wenn die Aufgabe nicht darin besteht, etwas zu tun oder zu machen, sondern schlicht darin zu erfassen, was der Fall ist?

Diese Fragen werfen vor einem Kantischen Hintergrund ein Rätsel auf, das sich auf die Anwendung von Begriffen wie „Aktivität“ und „Selbstbestimmung“ auf theoretische Erkenntnis bezieht. Ein ähnliches Rätsel stellt sich in gegenwärtigen Debatten bezüglich der Möglichkeit „doxastischer Tätigkeit“ [doxastic agency]. Überzeugungen scheinen nicht die Art von Einstellungen zu sein, die wir uns nach Belieben zueigen machen können: Denn haben wir einen zwingenden Grund für die Wahrheit einer gegebenen Proposition, scheint es unvermeidlich, diese Proposition zu glauben; sehen wir indessen keine Grundlage für die Wahrheit einer Proposition, dann scheint genau dieses Bewusstsein eine aufrichtige Überzeugung auszuschließen. Aber dann

1 Kant, Kritik der reinen Vernunft, A51/B75. Im Folgenden abgekürzt als KrV, A.d.Ü. 
scheint der Vorschlag, dass wir hinsichtlich unserer eigenen Überzeugungen tätig [agents] sind, bestenfalls undurchsichtig. Welche Art von Tätigkeit kann darin enthalten sein, einfach das Offensichtliche zu akzeptieren und das Nichtoffensichtliche als solches zu erkennen?

Doch trotz der Rätsel, die sie aufwirft, behält die Idee, dass wir eine Art der Kontrolle über unsere eigenen Überzeugungen ausüben können, ihre Anziehungskraft. So behandeln wir kognitiv voll entwickelte Menschen in einer Weise als verantwortlich für ihre eigenen Überzeugungen, die eine Art von Vermögen der kognitiven Selbstbestimmung vorauszusetzen scheint. Dies zeigt sich daran, dass wir für gewöhnlich dazu neigen, von einer Person zu verlangen, dass sie für die Dinge geradesteht, die sie glaubt - dass sie ihre Gründe für ihre Überzeugungen erläutert und sie gegen nachvollziehbare Kritik verteidigt. Wenn uns die Überzeugung, die eine Person in Hinsicht auf einen bestimmten Punkt bekundet, als nachweislich falsch oder schlecht begründet erscheint, ist es ferner so, dass wir von ihr verlangen, die Sache neu zu überdenken - und wir erwarten, dass ein erneutes Überdenken nicht bloß ihre Selbstbewertung, sondern ihre Überzeugungen erster Stufe ändert.

Kurzum, wir scheinen die Überzeugungen eines Subjekts als etwas zu betrachten, das in einer charakteristisch selbsttätigen [agential] Weise von ihm abhängt.

Könnten uns Kants Bemerkungen über die Spontaneität des Verstandes helfen, den Sinn zu klären, in dem vernünftige Subjekte in Hinsicht auf ihre Überzeugungen tätig sind? Ein zeitgenössischer Philosoph, der das denkt, ist John McDowell. Nach McDowell gilt:

Der Punkt der Idee, dass der Verstand ein spontanes Vermögen ist, besteht teilweise darin, dass das Netz [verknüpfter Vermögen], durch das ein einzelner Denker sein Denken bestimmt findet, nicht unantastbar ist. Aktives empirisches Denken erfolgt unter der ständigen Pflicht, über die Rechtfertigungsgrundlage der vermeintlich rationalen Verknüpfungen, die es bestimmen, nachzudenken. ${ }^{2}$

McDowell behauptet, dass sich die Spontaneität unseres Verstandes vorrangig in unserem Vermögen manifestiert, uns von den Überzeugungen und Schlüssen, zu denen wir tendieren, zu distanzieren, über solche Überzeugungen und Schlüsse „,nachzudenken“ und aktiv darüber zu „urteilen“, ob sie gültig sind. Zu urteilen ist dabei die Ausübung von Spontaneität par excellence, wie er behauptet:

2 McDowell (1994), 12 [dt. Übers. von J.Z.]. 
„Zu urteilen, das heißt zu entscheiden, was man denken soll, ist etwas, für das wir prinzipiell verantwortlich sind - etwas, das wir frei tun, im Unterschied zu dem, was uns in unserem Leben bloß widerfährt. Natürlich ist eine Überzeugung nicht immer, sogar nicht einmal üblicherweise, das Ergebnis unserer Ausübung dieser Freiheit zu entscheiden, was man denken soll. Aber selbst dann, wenn man nicht aus Freiheit zu einer Überzeugung gelangt, handelt es sich dennoch um die Ausübung von Fähigkeiten einer Art - nämlich des Begrifflichen -, deren paradigmatischer Modus der Aktualisierung im Gebrauch derjenigen Freiheit liegt, die im Urteilen besteht.“3

McDowell beruft sich im Zusammenhang seiner Verteidigung einer von Kant inspirierten Position in der Philosophie der Wahrnehmung auf Kants Begriff der Spontaneität und versucht nicht, seine Lesart von Kant detailliert anhand des Textes zu begründen. Angesichts der Wirkung von McDowells Diskussion lohnt es aber zu fragen, wie nahe seine Interpretation dem historischen Kant kommt. Tatsächlich will ich nahelegen, dass McDowells Erläuterung von der „Spontaneität des Verstandes“"Kants Idee beträchtlich verzehrt. Ich glaube, dass es lohnt, diese Fehldeutung zu korrigieren und zwar nicht nur um willen einer richtigen Lesart von Kant, sondern weil ich McDowells Überzeugung teile, dass zeitgenössische Philosoph_innen, die sich für doxastische Tätigkeit interessieren, immer noch von Kants Diskussion dieser Themen lernen können. Ich werde argumentieren, dass Kants Charakterisierung unseres Verstandes als spontan mit anderen Ansichten über die Natur des Erkennens verbunden ist, die auf interessante Weise von gegenwärtigen Annahmen abweichen, und dass eine Würdigung dieser Ansichten helfen kann, uns von einer Wahl zwischen den verschiedenen unbefriedigenden Alternativen zu befreien, die uns gegenwärtige Denkweisen aufzuzwingen scheinen.

\section{Exegetische Punkte}

Nach McDowells Lesart scheint die Spontaneität unseres Verstandes in Folgendem zu bestehen: Kraft des Vermögens, über unsere eigenen Einstellungen nachzudenken, können wir unsere eigenen Überzeugungen prüfen und uns fragen, ob sie berechtigt sind. Unsere Fähigkeit, unsere Überzeugungen in dieser Weise in Frage zu stellen, soll dabei insofern eine Art kognitiver Freiheit enthalten, als wir nicht einfach denjenigen Einstellungen ausgeliefert sind, die durch die natürlichen Tendenzen unseres kognitiven Systems in Kraft gesetzt werden. Und wenn wir tatsächlich über die Gründe für eine gegebene Einstellung nachdenken und ein Urteil fällen, dann soll dieses Urteil auf

3 McDowell (1998), 434 [dt. Übers. von J.Z.]. 
eine Weise ein $A k t$ des Subjekts sein, in der es bloß unreflektierte Überzeugungen nicht sind. Der spontane Charakter unseres Verstandes kommt damit nicht in unserem Überzeugtsein schlechthin zum Ausdruck, sondern in unserem Vermögen, über unsere eigenen Überzeugungen in einer bestimmten Weise nachzudenken und in Hinsicht auf sie tätig zu sein.

Diese Konzeption doxastischer Tätigkeit ist unter zeitgenössischen Philosophen weit verbreitet. Ich denke aber, dass sie nicht einfängt, was Kant vorschwebte, als er unseren Verstand als „spontan“ charakterisiert hat. Es wird sich als nützlich erweisen, zu Beginn zwei Merkmale von Kants Diskussion hervorzuheben, die darauf hindeuten, dass er etwas anderes vor Augen hatte. Erstens ist es nicht einfach, bei Kant ein großes Interesse an der Art von Urteilsakt zu finden, auf die McDowell den Fokus richtet. Es ist zwar richtig, dass Kant sagt, „[w]ir können aber alle Handlungen des Verstandes auf Urteile zurückführen““4, sodass man den Verstand als ein „Vermögen der Urteile ${ }^{65}$ charakterisieren kann, aber Der Begriff des Urteils, der zur Debatte steht, scheint nicht McDowells Begriff eines bewussten Ereignisses der „Entscheidung, was man denken soll“ zu sein, das eine Prozess des Überlegens abschließt und in einer Überzeugung „,resultiert“ ${ }^{6}$ Von Dingen, die ein Subjekt für wahr hält, spricht Kant ganz allgemein als seinem Urteilen, ganz egal, ob die relevanten Propositionen jemals Gegenstand bewussten Nachdenkens waren. Er spricht sogar von synthetischen Prinzipien a priori, die von jeglichem Verstandesvermögen immer schon als Urteile anerkannt sein müssen. ${ }^{7}$ Darüber hinaus definiert er Überzeugungen als eine Art des

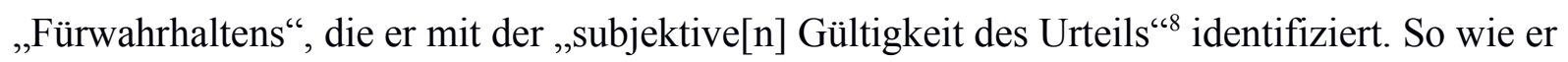
Urteile versteht, sind Überzeugungen also eine Spezies von Urteilen und zwar solche, die mit dem Bewusstsein verbunden sind, dass die eigenen Gründe für sie die Angelegenheit nicht objektiv entscheiden. Wenn Kant also vom „Urteilen“ spricht, scheint er nicht ein Ereignis kognitiver Bestätigung im Kopf zu haben, das Überzeugungen hervorbringt, sondern die Bedingung dafür, etwas als so-und-so aufzufassen und damit von Überzeugungen selbst. Soweit ich weiß, gibt es bei Kant keinen Hinweis darauf, dass unser Vermögen, von unseren Überzeugungen ,zurückzutreten“, der spezielle und paradigmatische Ausdruck der Spontaneität unseres Verstandes ist. Diese Spontaneität kommt direkt in unserem Erkennen selbst zum Ausdruck: in unseren Überzeugungen oder im günstigen Fall in unserem Wissen, dass die Dinge so-und-so sind.

Zweitens gibt es einen wichtigen Aspekt von Kants Charakterisierung der Spontaneität

\footnotetext{
$4 \mathrm{KrV}, \mathrm{A} 69 / \mathrm{B} 94$.

$5 \mathrm{KrV}, \mathrm{A} 126$.

6 Für ähnliche Beobachtungen siehe Engstrom (2009), 103-104.

7 Vgl. z.B. KrV, B14-18, A148/B187.

$8 \mathrm{KrV}, \mathrm{A} 822 / \mathrm{B} 850$.
} 
des Verstandes, die McDowells Lesart nicht erklärt. Kant sagt mehrfach, dass der spontane Charakter des Verstandes hauptsächlich darin zum Ausdruck kommt, dass er Akte der Synthesis vollzieht, wobei Synthesis als die „Handlung, verschiedene Vorstellungen zu einander hinzuzutun, und ihre Mannigfaltigkeit in einer Erkenntnis zu begreifen“99 verstanden wird. Ein Urteil zählt deshalb als spontaner Akt, weil es die Vorstellung der Verbindung einer Mannigfaltigkeit von Begriffen ist und weil diese Vorstellung einer Verbindung von einem Akt der Synthesis abhängt. ${ }^{10}$ Es ist nicht klar, was McDowell im Rahmen seiner Lesart aus diesen Behauptungen machen kann. Der Gedanke, dass die Spontaneität des Verstandes grundlegend in dessen Vermögen besteht, eine Mannigfaltigkeit zu synthetisieren, hat kein eindeutiges Gegenstück in seiner Interpretation. Diese richtet ihren Fokus nicht auf unser Vermögen, Vorstellungen in einer Einheit zu verbinden, sondern auf unser Vermögen, über ganze Propositionen nachzudenken und diese einer Art von Ja-oder-Nein Votum zu unterziehen.

\section{Substanzielle Zweifel}

Bis hierhin habe ich exegetische Gründe für meine Zweifel angeführt, dass McDowells Erläuterung unserer kognitiven Spontaneität Kants Idee einfängt. Nun will ich mich einigen substanziellen Zweifeln darüber zuwenden, ob McDowells Erläuterung wirklich den Sinn erfasst, in dem wir in Hinsicht auf unsere eigene Erkenntnis tätig sein können.

Meine Zweifel beziehen sich auf die Verbindung, die McDowells Position zwischen dem Ereignis einführt, das meine eigentlich Ausübung von Tätigkeit sein soll - meinem Fällen eines Urteils -, und dem Überzeugungszustand, der aus dieser Aktivität resultieren soll. ${ }^{11}$ McDowell macht nicht explizit, wie wir diese Verknüpfung zu verstehen haben. Er spricht davon, dass eine Überzeugung aus einem Urteil „,resultiert“, es ist aber nicht klar, welche Art von Verknüpfung hier gemeint ist (ich werde gleich einige Möglichkeiten nennen). So viel ist allerdings klar: Seiner Ansicht nach darf meine Ausübung von Tätigkeit nicht mit meinem Überzeugungszustand selbst gleichgesetzt werden. Wenn dem nämlich so wäre, dann wäre es nicht nötig zuzugestehen, dass eine Überzeugung, obwohl sie ,nicht immer, sogar nicht einmal üblicherweise, das Ergebnis unserer Ausübung [unserer] Freiheit zu entscheiden [ist], was man denken soll“, dennoch eine „Ausübung von Fähigkeiten einer Art - nämlich des Begrifflichen - [darstellt], deren paradigmatischer Modus der Aktualisierung im Gebrauch derjenigen Freiheit liegt, die im Urteilen besteht"“.

McDowell ist nicht der einzige, der Überzeugungen und Urteile auf diese Weise

$9 \mathrm{KrV}, \mathrm{A} 77 / \mathrm{B} 103$.

10 Vgl. KrV, B129-130, A68/B93, A79/B105.

11 Diese Art von Kritik wird detaillierter in Boyle (2011a) und (2011b) entwickelt. 
unterscheidet. Viele zeitgenössische Philosophen behaupten, dass Überzeugungen nicht-aktive Zustände, Urteile hingegen aktive Ereignisse sind. Um nur ein Beispiel zu nennen, hier ist, was Sydney Shoemaker sagt:

„Etwas zu glauben - d.h. die fortwährende Überzeugung zu haben, dass das und das der Fall ist - ist kein Akt. Zu urteilen, verstanden als mentales Vorkommnis und nicht als fortwährender Zustand, ist hingegen ein Akt.“"12

Was Autoren vorschwebt, die auf diese Weise sprechen, sollte uns in meinen Augen obskur anmuten. Was soll dieser Akt des Urteils sein? Ich kann in der Tat innerlich durchsprechen, was ich als Wahrheit bezüglich irgendeiner Sache betrachte und mir bloß vorstellen, welche Worte ich aussprechen würde, wenn ich meine Meinung laut verkünden würde. Ich bin bereit zuzugestehen, dass eine solche innere Selbstartikulation ein freiwilliger Akt sein kann: Ich kann mir zu meinem Vergnügen die Aufgabe stellen, meine Ansichten bezüglich eines Punktes innerlich durchzusprechen, genauso wie ich mir die Aufgabe stellen kann, meine Ansichten laut zu verkünden. Ein Urteilsakt soll aber vermutlich einen entschiedeneren Charakter haben als das: In einem Urteil bestätige ich mir nicht bloß, was ich glaube, sondern lege meine Überzeugung festich „entscheide, was man denken soll“, wie McDowell es ausdrückt. In welchem Sinn könnte dies aber ein Akt sein, wenn Überzeugungen selbst nicht aktiv sind?

Um das Problem hier zu sehen, sollten wir über die Frage nachdenken, ob zu urteilen, dass $\mathrm{p}$, selbst schon voraussetzt, dass man p glaubt. Wenn zu urteilen, dass $\mathrm{p}$, die Überzeugung, dass $\mathrm{p}$, voraussetzt - wenn ich also in dem Moment, in dem ich urteile, dass $\mathrm{p}$, glauben muss, dass $\mathrm{p}-$, dann scheint es so, als könnte ich meine Überzeugung bezüglich p nicht festlegen, indem ich urteile, dass p, denn mein Urteilsakt würde voraussetzen, dass dieser Zustand besteht. Dass ich, nachdem ich überlegt habe, ein Urteil fälle, wäre in diesem Fall der erste bewusste Ausdruck einer neu geformten Überzeugung. Es könnte sich aber nicht um einen Akt handeln, durch den ich eine Überzeugung in mir hervorbringe. Ich tue nichts, das in meiner Überzeugung resultiert. Der Akt steht zu meiner Überzeugung nicht im Verhältnis von Mittel zu Zweck oder von Ursache zu Wirkung, sondern im Verhältnis von Ausdruck zur ausgedrückten Bedingung.

Wie verhält es sich mit der anderen Alternative, wonach zu urteilen, dass p, nicht voraussetzt, dass man p glaubt? Ich denke, dass uns die Idee, ich könnte urteilen, dass p, ohne zu glauben, dass p, als schwer verständlich anmuten sollte. Denn zu urteilen, dass p, verlangt sicherlich

\footnotetext{
12 Shoemaker (2009), 36 [dt. Übers. von J.Z.].
} 
mehr, als bloß zu denken oder innerlich zu sagen, dass $p$. Wenn ich die Idee, dass $p$ der Fall sein könnte, bloß unverbindlich erwäge oder wenn ich ohne es zu bekräftigen innerlich sage, dass $p$, dann habe ich damit nichts bezüglich dessen entschieden, ob ich die Wahrheit von $p$ akzeptiere, und damit habe ich vermutlich auch nicht geurteilt. Zu sagen, dass ich urteile, dass p, enthält mit Sicherheit, dass ich die Wahrheit von p bekräftige. Es ist aber schwer zu begreifen, wie das etwas anderes meinen kann, als dass mein Urteil meine Überzeugung, dass p, enthalten muss. Folglich ist es schwer zu begreifen, wie ich urteilen kann, dass p, ohne p zu glauben.

Ich denke, die Anziehungskraft der Idee, dass zu urteilen ein Akt der Entscheidung darüber ist, was man denken soll, beruht zu großen Teilen auf einer Vermischung zweier unterschiedlicher Ideen: der unproblematischen Idee eines Akts, der darin besteht, innerlich durchzusprechen, was man glaubt; und der wohl inkohärenten Idee, man könne eine Überzeugung in sich hervorbringen, indem man eine Proposition als wahr bestätigt. Etwas innerlich durchzusprechen, ist ein vollkommen geeignetes Beispiel für einen Akt, aber keine Ausübung kognitiver Tätigkeit: man wirkt hierdurch nicht auf die eigene kognitive Verfassung ein, sondern bringt diese bloß zum Ausdruck. Philosophen sprechen oftmals so, als würde es eine andere Art von Akt geben - „urteilen“ -, der in einer Ausübung von kognitiver Tätigkeit besteht - einen Akt, durch den man festlegt, was man glaubt, oder eine Überzeugung in sich selbst hervorbringt. Wenn wir aber sorgfältig darüber nachdenken, worin dieser Akt bestehen soll, dann kollabiert diese Idee entweder in das innere Durchsprechen dessen, was man glaubt, oder sie zerfällt in Inkohärenz.

Selbst wenn wir um des Arguments willen zugestehen, dass ein Urteilsakt eine Überzeugung hervorbringen kann, sollten wir diese Konzeption von doxastischer Tätigkeit, meiner Meinung nach, unbefriedigend finden. Wenn wir mit McDowell und Shoemaker darin übereinstimmen, dass Urteilen ein Akt ist, von etwas überzeugt zu sein aber nicht, dann können wir die Überzeugung, dass p, bestenfalls als Resultat doxastischer Tätigkeit begreifen. Es folgt, dass der Sinn, in dem ich meinen kognitiven Zustand kontrolliere, wenn ich bewusst urteile, ein ganz anderer ist als der, in dem ich meinen kognitiven Zustand kontrolliere, wenn ich bloß eine bestimmte Überzeugung habe. Im ersteren Fall übe ich mein Vermögen, mich kognitiv selbst zu bestimmen, tatsächlich aus. Im letzteren kontrolliere ich meine Situation bloß potentiell, insofern ich die fragliche Überzeugung neu überdenken kann. Die Frage, auf die ich pochen will, lautet: Haben wir es hier mit der richtigen Art von Verantwortung für das zu tun, was wir glauben, d.h. mit der Art von Verantwortung, deren intuitives Verständnis die Idee nahelegt, dass unsere Überzeugungen von uns selbst abhängen?

Vergegenwärtigen wir uns einige der üblichen Weisen, in denen wir Personen als 
verantwortlich für ihre eigenen Überzeugungen betrachten. Von einer Person, die p glaubt, erwarten wir normalerweise, dass sie die Frage, warum sie p glaubt, beantworten kann und zwar unabhängig davon, ob sie darüber jemals bewusst nachgedacht hat. ${ }^{13}$ Eine Person, die mit solch einer Frage konfrontiert ist, hat natürlich nicht immer spezifische Gründe für eine gegebene Überzeugung. Das Interessante ist aber Folgendes: Selbst wenn jemand zugibt, dass es ihm an Gründen mangelt, akzeptiert er, was die Frage voraussetzt - nämlich dass er in der Position ist, seine Gründe zu äußern, welche auch immer das sind. Weiterhin gilt: Wenn eine Person Gründe vorbringt, die offenkundig dürftig sind oder wenn sie zugibt, in einem Fall keine Gründe zu haben, in dem es offensichtlich Gründe braucht, sind wir geneigt, ihre Überzeugung zu kritisieren. Dabei ist von großer Bedeutung, dass wir unsere Kritik an sie richten. Wir fragen sie, warum sie etwas derartig Seltsames denkt, wie sie solch ein offensichtlich unvernünftiges Argument akzeptieren kann, etc. Kurzum scheint es so, als würden wir schlicht den Umstand, dass eine Person eine Überzeugung hat, als Ausdruck ihres Vermögens begreifen, das, was sie glaubt, zu bestimmen, indem sie prüft, ob eine bestimmte Proposition wahr ist vor dem Hintergrund von Gründen, die sie als relevant erachtet - und wir tun dies, ganz egal ob die Person jemals über die fragliche Überzeugung nachgedacht hat. Kann McDowells Interpretation kognitiver Tätigkeit dies erklären? Ausgehend von seiner Position besteht die Schwierigkeit darin zu begreifen, warum wir eine Person, die ohne nachzudenken von etwas überzeugt ist, als verantwortlich für ihre Überzeugung betrachten sollten. So lautet die Hypothese doch gerade, dass sich in ihrer Überzeugung keine wirkliche Ausübung doxastischer Tätigkeit spiegelt. Könnte McDowell entgegnen, dass wir, selbst wenn wir p ohne zu überlegen glauben, in Hinsicht auf diese Überzeugung jederzeit dadurch tätig werden können, dass wir zu überlegen beginnen, ob p? Es ist schwierig zu sehen, wie dieser Punkt die Tatsachen erklären kann, die wir hervorgehoben haben. Mir wird nicht bloß die Verantwortung dafür zugewiesen, dass ich einer unvernünftigen Überzeugung erlaube, eine meiner Überzeugungen zu bleiben; vielmehr werde ich als verantwortlich dafür betrachtet, dass ich diese Überzeugung jetzt habe - dafür, dass ich im Kontext der Gründe, die mir zur Verfügung stehen, die Dinge gegenwärtig als so-und-so begreife. Von mir wird erwartet, die Frage, warum ich gegenwärtig diese Überzeugung habe, beantworten zu können, und ich werde als verantwortlich für die Antwort betrachtet, selbst wenn ich niemals über die Angelegenheit nachgedacht habe. Damit scheinen wir den Umstand, dass eine Person eine unreflektierte Überzeugung hat, nicht bloß als etwas zu behandeln, auf das sie potentiell Einfluss ausüben kann, sondern vielmehr als etwas, in dem ihr Vermögen, sich kognitiv

13 Wir erwarten das von einem Subjekt, das darüber nachdenken kann, was der Fall ist und welche Behauptungen glaubwürdig sind. Meine Behauptungen im Rahmen dieser Diskussion sollten durchgängig so verstanden werden, dass sie nur auf Subjekte zutreffen, die diese Vermögen besitzen. 
selbst zu bestimmen, bereits tätig ist. Ich komme zu dem Schluss, dass McDowells Lesart unsere Tätigkeit an einem Punkt verortet, der unserer kognitiven Verfassung zu äußerlich ist, um den Charakter unserer Verantwortung gegenüber dem zu erklären, wovon wir überzeugt sind.

\section{Eine andere Interpretation}

Wir haben gesehen, dass McDowells Lesart problematisch ist und zwar sowohl als eine Interpretation von Kants Aussagen zur Spontaneität des Verstandes als auch im Sinne einer Erklärung der Art von Tätigkeit, die wir im Erkennen ausüben. Wie müssen wir Kants Behauptung, dass unser Verstand spontan ist, aber verstehen, wenn wir McDowells Lesart zurückweisen? Henry Allison hat eine andere Lesart skizziert ${ }^{14}$ und ich denke, Allisons Interpretation weist uns den richtigen Weg - auch wenn sie in einigen wichtigen Hinsichten, wie wir sehen werden, unbefriedigend bleibt.

Wenn Kant den Verstand als spontan charakterisiert, sollten wir seinen Punkt gemäß Allison im Sinne der Idee verstehen, dass der Verstand ein Vermögen ist, "etwas als etwas aufzufassen" [taking as], wobei $\mathrm{x}$ als ein $\mathrm{F}$ aufzufassen unter folgende Bedingung fällt: "[W]enn man sich nicht bewusst ist, $\mathrm{x}$ als $\mathrm{F}$ aufzufassen (es in einem Begriff zu erkennen), hat man es tatsächlich nicht als solches aufgefasst. “15

Und allgemeiner: Wann immer man etwas als so beschaffen auffasst oder als etwas, das aus etwas anderem folgt, muss man sich dessen bewusst sein. Allison nennt diese Bedingung Kants Apperzeptionsbedingung (AB) und denkt, sie fange Kants Idee ein, dass die charakteristischen Akte unseres Verstandes - Begreifen [conceiving], Urteilen und Schließen - "inhärent selbstbewusst" sind. Hieraus folgt, wie er behauptet, dass unser Bewusstsein dieser Aktivitäten nicht "in zwei distinkte Aktivitäten zergliedert werden kann, nämlich ein Vorstellen und ein Vorstellen, dass man vorstellt. Sich seiner Aktivität bewusst zu sein, ist keine andere Sache, die man zusätzlich zum Urteilen oder Schließen tut; es handelt sich vielmehr um einen nicht eliminierbaren Bestandteil der Aktivität erster Stufe selbst."16

Allison argumentiert weiterhin, (AB) impliziere, dass man Begreifen, Urteilen und Schließen als spontane Akte verstehen muss:

„Gerade weil die Anerkennung [des eigenen Urteils] nicht einfach als weitere Überzeugung verstanden werden kann [...], ist sie nichts, das man kohärenterweise als

14 Vgl. Allison (1996a), (1996b).

15 Ders. (1996a), 95 [dt. Übers. von J.Z.].

16 Ebd. [dt. Übers. von J.Z.]. 
etwas verstehen kann, das einem bloß widerfährt [...]. Weil diese [Anerkennung] kein kognitiver Zustand ist, in dem das Subjekt sich vorfindet, sondern ein Akt, den es für sich selbst (selbstbewusst) vollziehen muss, [...] müssen wir von einer absoluten und nicht bloß relativen Spontaneität ausgehen, um seine Möglichkeit zu begreifen. “17

Bevor wir diese Erläuterung der Spontaneität des Verstandes im Detail prüfen, möchte ich innehalten, um zwei Unterschiede zu McDowells Position hervorzuheben. Zum einen haben wir gesehen, dass McDowells Lesart Kants Idee, dass unser Verstand wesentlich ein Vermögen ist, Vorstellungen zu synthetisieren, nicht erklärt. Im Unterschied dazu zielt Allisons Vorschlag, dass jede Erkenntnis damit einhergeht, dass „etwas als etwas aufgefasst“" wird, gerade darauf ab, diese Idee einzufangen. Allisons Vorschlag lautet, dass unser Verstand eine Mannigfaltigkeit von Vorstellungen (besondere Vorstellungen, die unter einem Begriff vereint sind; Begriffe, die in einem Urteil vereint sind; Urteile, die in einem Schluss verknüpft sind) in einer Erkenntnis vereint, indem er die relevanten Vorstellungen so auffasst, dass sie in einer bestimmten Weise verknüpft sind. Die Idee, dass dieses „Auffassen“ (AB) untersteht, ist Allisons Erläuterung von Kants Behauptung, dass ich, um eine Mannigfaltigkeit von Vorstellungen zu verknüpfen, diese Vorstellungen synthetisieren und mir des Akts der Synthesis bewusst sein muss. ${ }^{18}$

Es gibt weiterhin einen Unterschied in Hinsicht auf die Rolle, die beide Lesarten dem Selbstbewusstsein zuschreiben. Bei McDowell erlaubt uns unser Bewusstsein von unseren eigenen kognitiven Zuständen eine Art von Tätigkeit in Hinsicht auf diese auszuüben, weil es uns erlaubt, explizit über ihre Gültigkeit nachzudenken und sie gemäß unserem Urteil abzuändern. Im Kontrast dazu besteht die vorrangige Bedeutung des Selbstbewusstseins gemäß Allisons Lesart nicht darin, dass es uns erlaubt, von unseren kognitiven Haltungen „zurückzutreten“ und sie zu verändern, sondern darin, dass es in die Konstitution dieser Haltungen mit einfließt. Wenn zu urteilen eine Art davon ist, etwas als etwas aufzufassen, und wenn etwas als etwas aufzufassen (AB) untersteht, dann ist ein Urteil, von dem ein Subjekt kein Bewusstsein hat, überhaupt kein Urteil.

Ich habe zuvor dafür argumentiert, dass Kant den Ausdruck „Urteil“ nicht für eine besondere Art von Ereignis verwendet, das Überzeugungen hervorbringt, sondern für eine fortwährende kognitive Haltung: etwas für so beschaffen halten oder im günstigen Fall wissen, dass es so beschaffen ist. Mir ist nicht klar, ob Allison Kants Rede von Urteilen auf diese Weise liest. Einige seiner Bemerkungen legen nahe, dass er die zeitgenössische Konzeption von Urteilen im

17 Ders. (1996b), 64 [dt. Übers. von J.Z.].

18 Vgl. KrV, B133, B141-142, B153. 
Sinn hat, wonach ein Urteil ein bewusstes Ereignis ist, das (manche) Überzeugungen hervorbringt. Trotzdem hängt nichts in der Struktur von Allisons Vorschlag von dieser Konzeption des Urteils ab und wenn wir sie beiseite stellen, eröffnen wir Raum für eine Konzeption unseres Erkennens als aktiv und zwar nicht nur bei solchen Gelegenheiten, in denen wir bewusst überlegen, sondern wann immer wir (in fortwährender Weise) die Dinge als so-und-so auffassen.

Es gilt zu beachten, wie diese Konzeption unserer Aktivität im Erkennen die Schwierigkeiten vermeidet, die ich für McDowells Position aufgeworfen habe. Zum einen umgeht sie das Dilemma, das sich aus unserer Frage ergeben hat, ob Urteile Überzeugungen voraussetzen, und zwar weil sie Urteile nicht als Akte versteht, die Überzeugungen hervorbringen bzw. in diesen resultieren. Allisons Interpretation lokalisiert unsere kognitive Tätigkeit nicht in Ereignissen der bewussten Bejahung einer Proposition, sondern in der zugrunde liegenden Haltung des Auffassens von etwas als etwas, die solche Ereignisse ausdrücken können. Allison muss also nicht behaupten, dass die paradigmatische Ausübung unserer kognitiven Spontaneität im Urteil liegt und dass Überzeugungen nur in einem untergeordneten Sinn als frei gelten, nämlich kraft des Umstands, dass sie „Ausübungen von Fähigkeiten sind, deren paradigmatischer Modus der Aktualisierung im Urteil besteht“، Gemäß seiner Lesart kann die Bedingung von Überzeugungen selbst als eine Ausübung unserer kognitiven Spontaneität gelten und zwar insofern sie beinhaltet, dass etwas als so-und-so aufgefasst wird. Und aus dem gleichen Grund vermeidet diese Interpretation den intuitiven Einwand, dass unsere Tätigkeit an einem Punkt lokalisiert wird, der unserer kognitiven Verfassung zu äußerlich ist, um unsere Verantwortung für das, was wir für wahr halten, zu erklären. Denn sie lokalisiert unsere Aktivität gerade darin, dass wir eben diese Einstellung haben.

Die Schwierigkeit besteht nun natürlich darin, die Idee zu erhellen, dass unser Erkennen wesentlich selbstbewusst und damit seinem Charakter nach aktiv ist und das nicht bloß in Momenten, in denen wir bewusst nachdenken, sondern immer dann, wenn sich unser Vermögen zu wissen in einem aktualisierten Zustand befindet. Ich denke nicht, dass Allisons Erklärung dieser Dinge zufriedenstellend ist. Erstens ist weder die Natur von (AB) noch die Motivation für (AB) hinreichend klar. Es fällt leicht zu sagen, dass eine Person x nur dann als F ,,auffasst“", wenn sie sich bewusst ist, dass sie $\mathrm{x}$ als F auffasst. Aber um welche Art von Bewusstsein geht es hier? Die relevante Art des „Auffassens“ soll, wie wir gesehen haben, keine zusätzliche, distinkte Vorstellung von sich als jemand enthalten, der x als F auffasst. In der Tat gilt: Wenn (AB) einen Hauch von Plausibilität als generelle Bedingung des Erkennens haben soll, dann muss ein derartiges „Auffassen“ auch dann möglich sein, wenn ein Subjekt in keiner Weise mit seiner eigenen kognitiven Aktivität befasst ist, sondern sich vollständig auf das fokussiert, was auch immer es zu 
verstehen versucht. An dieser Stelle können wir aber ein Dilemma in Hinsicht auf die Interpretation von (AB) aufwerfen. Wenn der Anspruch auf Selbstbewusstsein auf der einen Seite zu substanziell gestaltet wird, dann droht (AB) eine unplausible Bedingung des Erkennens zu werden und es wird sich als schwierig erweisen, $(\mathrm{AB})$ mit der Idee in Einklang zu bringen, dass ein solches Bewusstsein keine zusätzliche, distinkte Vorstellung erfordert. Wenn der Anspruch auf der anderen Seite nicht substanziell genug gestaltet wird, dann wird es schwierig zu sehen, was (AB) überhaupt bedeuten soll.

Es besteht auch eine Schwierigkeit in Hinsicht auf das Argument, das die Behauptung, dass Urteile $(\mathrm{AB})$ unterstehen, mit der Idee verbindet, dass Urteile spontane Akte sind (nennen wir diese Idee die Spontaneitätsthese (ST)). Allison scheint wie folgt zu argumentieren: ${ }^{19}$

i. Wenn ein Subjekt S auf der Grundlage irgendeines Grundes G urteilt, dass p, dann muss sich $S$ gemäß (AB) bewusst sein, p aufgrund von $G$ für wahr zu halten.

ii. Wenn S' Urteil, dass p, kausal durch die vorhergehenden mentalen Zustände von S bestimmt ist, dann kann S' Urteil, dass p, nicht von S' selbstbewusstem Akt abhängen, p aufgrund von $\mathrm{G}$ für wahr zu halten.

iii. Also: Wenn S auf Grundlage irgendeines Grundes G urteilt, dass p, dann kann S' Urteil, dass p, nicht kausal durch seine vorgehenden mentalen Zustände bestimmt sein.

iv. Wenn S'Urteil, dass p, nicht kausal von seinen vorhergehenden mentalen Zuständen bestimmt ist, dann ist sein Urteil ein spontaner Akt.

v. Also: Jeder Akt, in dem ein Subjekt auf Grundlage irgendeines Grundes G urteilt, dass p, muss ein spontaner Akt sein.

Es gibt verschiedene Punkte in diesem Argument, die ich gerne erörtern würde, aber hier werde ich den Fokus auf ein zentrales Bedenken richten. Gestehen wir zu, dass (AB) Folgendes beinhaltet: Wenn S aufgrund von G urteilt, dass p, dann muss sich S des Umstands bewusst sein, dass es p aus diesem Grund für wahr erachtet. Gestehen wir weiterhin zu, dass dieses Selbstbewusstsein keine zusätzliche, distinkte Vorstellung, sondern eine notwendige Bedingung dafür ist, dass S das relevante Urteil überhaupt fällt. Warum könnte dieser gesamte Sachverhalt - der Sachverhalt, dass S p aufgrund von $\mathrm{G}$ in selbstbewusster Weise als wahr erachtet - keiner sein, der kausal durch $\mathrm{S}^{\prime}$ vorhergehende mentale Zustände bestimmt ist?

19 Was folgt ist eine Rekonstruktion einer Art von Argument, das Allison in Allison (1996a), 100-104 und (1996b), 6364 skizziert. In seiner Diskussion wechselt Allison zwischen der Rede von Überzeugungen und Urteilen, aber soweit ich sehen kann, hängt nichts davon ab. Ich formuliere das Argument durchgängig in Bezug auf Urteile. 
Allison erzeugt hier den Eindruck eines Widerspruchs, indem er annimmt, dass die eigenen Gründe (bestimmte) vorhergehende eigene mentale Zustände sein müssen und dass es für das eigene Urteil, dass p, nicht notwendig sein kann, diese Zustände in einem selbstbewussten Akt als Gründe für das Urteil zu begreifen, wenn die bloße Gegenwart dieser Zustände für das Urteil hinreichend ist. Hier wird allerdings eine Vermengung zwischen der rechtfertigenden Rolle der Gehalte, die die eigenen mentalen Zustände aufweisen, und der kausalen Rolle dieser Zustände selbst ausgenutzt. Die Gehalte von verschiedenen meiner mentalen Zustände können mir Gründe geben zu urteilen, dass p, und ich kann - sofern (AB) gilt - aus diesen Gründen nur urteilen, wenn ich sie in einem selbstbewussten Akt als Gründe für mein Urteil begreife. Aber diese Anforderung enthält nicht, dass ich die kausale Geschichte meines Urteils gutheißen oder auch nur kennen muss: sie verlangt nur, dass ich weiß, dass ich ausgehend von den und den Gründen urteile, dass p, und nicht, dass ich weiß, dass mein Urteil durch mentale Zustände verursacht wurde, deren Gehalte diese Gründe zum Ausdruck bringen. Es gibt also keine Spannung zwischen der Idee, dass bestimmte vorhergehende mentale Zustände ein gegebenes Urteil kausal bestimmen und der Behauptung, dass dieses Urteil ein Bewusstsein der Gründe voraussetzt, die diese Zustände (durch ihre Gehalte) verfügbar machen. Es fehlt uns also an einem stichhaltigen Argument von (AB) zu (ST).

In jedem Fall scheint es mir so, dass Allisons Argument wenig Aufschluss über den Sinn gibt, in dem wir aktiv sind, wenn wir urteilen. Allison behauptet, dass es eine enge Verbindung zwischen der Idee gibt, dass zu urteilen ein Bewusstsein des Urteils voraussetzt und der Idee, dass zu urteilen ein aktives Phänomen ist. Ich teile diese Intuition, aber die Verbindung bleibt ziemlich unklar. Warum sollte ein wesentlich selbstbewusster mentaler Zustand oder ein wesentlich selbstbewusstes mentales Ereignis in irgendeinem interessanten Sinn ein Akt des Subjekts sein?

\section{Kantische Gedanken zum „Urteil“6}

Die Ergebnisse unserer Betrachtung von Allisons Interpretation sind durchmischt. Auf der einen Seite haben wir gesehen, dass seine Erläuterung dessen, was Kant mit der „Spontaneität des Verstandes" meint, vielversprechend ist und zwar sowohl im Sinne einer Auslegung Kants als auch substanziell, indem sie die Schwierigkeiten vermeidet, mit denen McDowells Position konfrontiert ist. Auf der anderen Seite fehlt es uns nach wie vor an einer verständlichen Erklärung davon, was es mit $(\mathrm{AB})$ auf sich hat, und wie (AB) mit der Idee verbunden ist, dass wir in unserem Erkennen aktiv sind. In diesem letzten Abschnitt will ich die Aufmerksamkeit auf einige Aspekte von Kants Konzeption des Urteils lenken, die mir diese Angelegenheiten zu erhellen scheinen. 
Wir können beginnen, indem wir einen Unterschied zwischen Kants Konzeption des Urteils und der zeitgenössischen Standardkonzeption beachten. Wenn zeitgenössische Philosophen von „urteilen“" sprechen, meinen sie gewöhnlich einen positiven oder negativen Urteilsspruch in Hinsicht auf eine Proposition: entweder man bejaht, dass p, oder man unterlässt es, $\mathrm{p}$ zu bejahen. Wenn Kant dagegen von „urteilen“ spricht, dann hat er typischerweise etwas im Sinn, das er die „Erweiterung“20 einer gegebenen Erkenntnis eines Gegenstands durch die Hinzufügung einer weiteren Erkenntnis nennt. Diese Charakterisierung trifft am eindeutigsten auf synthetische kategorische Urteile zu, in denen wir ein Prädikat so mit einem Subjekt verbinden, dass wir eine neue Erkenntnis hervorbringen, indem wir unseren bestehenden Begriff eines Gegenstands weiter „bestimmen“. Kant meint aber, dass diese Struktur in einer abgeleiteten Weise auch auf hypothetische und disjunktive Urteile zutrifft: auch diese können eine gegebene Erkenntnis erweitern und lassen sich damit - in einem weiten Sinne - als Weisen verstehen, von einem gegebenen „Subjekt“ eine weitere Bestimmung zu ,prädizieren“. ${ }^{21}$ Diese allgemeine Idee über das Urteil - dass es von einer gegebenen Erkenntnis ausgeht und mit ihrer weiteren Bestimmung fortfährt - ist in Kants Charakterisierung unserer Erkenntnis als „diskursiv“ enthalten, ein Ausdruck, dessen Kernbedeutung „,von einem Ding zu einem anderen übergehen“ lautet. ${ }^{22}$ Sich klar zu machen, dass Kant das Urteil in dieser Weise betrachtet hat, ist in meinen Augen der Schlüssel, um zu begreifen, warum er das Urteil als einen Akt der Synthesis charakterisiert. Wenn wir zu urteilen im Sinne der modernen Konzeption als Zustimmung zu einer Vorstellung begreifen, deren Einheit gegeben ist, dann werden wir den eigentlichen Punkt der Idee, dass zu urteilen eine Synthesis beinhaltet, nicht verstehen. ${ }^{23}$ Tatsächlich gründet die moderne Auffassung des Urteils auf der bewussten Zurückweisung der Konzeption, die Kant befürwortet hat. So attackiert Frege in seinem Aufsatz „Die Verneinung“ bekanntlich „die Meinung, der Urteilende stifte durch sein Urteil den Zusammenhang, die Ordnung der Teile und bringe dadurch das Urteil zustande ${ }^{624}$ und zwar mit folgender Begründung:

$20 \mathrm{KrV}, \mathrm{B} 10 / \mathrm{A} 7, \mathrm{vgl} . \mathrm{KrV}, \mathrm{B} 93 / \mathrm{A} 68$.

21 Für eine aufschlussreiche Diskussion dieses Punkts siehe Longuenesse (1998), Kapitel 4.

22 Man vergleiche die Diskussion in Engstrom (2006), 12. Für einen Beleg der Geschichte dieser Art von Verständnis in Hinsicht auf die „Diskursivität“, die den menschlichen Intellekt auszeichnet, siehe Thomas Aquin, Summa Theologica, I.14.7 and 1.58.4.

23 Die fregeanische Konzeption des Urteils wird in zeitgenössischen Diskussionen von Kants Theorie des Urteils oftmals stillschweigend vorausgesetzt. Für eine außergewöhnliche und zum Denken anregende Diskussion von Alternativen zur fregeanischen Konzeption siehe Kimhi (im Erscheinen).

24 Frege (1919), 74. 
„Jahre mühevoller Untersuchungen können zwischen dem Fassen des Gedankens und der Anerkennung seiner Wahrheit liegen. Daß durch dieses Urteilen der Gedanke, der Zusammenhang seiner Teile nicht gestiftet werde, ist offenbar; denn er bestand schon vorher. Aber auch das Fassen eines Gedankens ist nicht ein Schaffen des Gedankens [...] denn der Gedanke war schon vorher wahr, bestand also schon in der Ordnung seiner Teile, bevor er gefasst wurde. ${ }^{(25}$

Unser Begriff einer Proposition ist ein Nachkomme von Freges Begriff eines Gedankens und wir gehen - so wie Frege es getan hat - gewöhnlich davon aus, dass eine solche Entität als ein Ganzes existiert und bloß darauf wartet, von uns gefasst und vielleicht als wahr erkannt zu werden. Ich kann hier nicht auf die Vielzahl von Argumenten eingehen, die Frege für diese Auffassung des Urteils anführt, aber ich möchte Folgendes anmerken: Es ist nicht offensichtlich, dass sein Punkt bezüglich der vorher gegebenen Einheit dessen, was im Urteil gewusst (als gewusst betrachtet) wird, unvereinbar mit Kants Konzeption der Art von Wissen ist, die das Urteil enthält. Gestehen wir zu, dass der mögliche Sachverhalt, dessen Wirklichkeit wir beurteilen müssen, nicht durch den Urteilsakt konstituiert wird; trotzdem könnte es wahr sein, dass unser Erkennen von etwas, das bereits verstanden ist, sich zu einem Verständnis wird entwickelt, das darüber hinausgeht und bestimmter ist. Kant begreift die Subjekt-Prädikat Struktur unserer grundlegenden Urteile als etwas, in dem sich dieser fortschreitende Charakter endlicher Erkenntnis spiegelt und er betrachtet die Aufgabe der „Bestimmung“ von Urteilen als eine, die - sozusagen - darin besteht, den weiteren Weg zu gestalten, indem man eine gegebene Erkenntnis mit einer neuen Bestimmung verbindet. Kants Gründe für die Behauptung, dass Urteile Akte und dass diese Akte selbstbewusst sind, stützen sich auf seine Ansichten bezüglich der Natur einer derartigen Verbindung. Wie weithin bekannt, behauptet Kant, dass

„die Verbindung (coniunctio), eines Mannigfaltigen überhaupt, [...] niemals durch Sinne in uns kommen [kann] [...]; denn sie ist ein Actus der Spontaneität der Vorstellungskraft [...] [Diese] Verstandeshandlung [...] [belegen] wir mit der allgemeinen Benennung Synthesis [...], um dadurch zugleich bemerklich zu machen, daß wir uns nichts, als im Objekt verbunden, vorstellen können, ohne es vorher selbst verbunden zu haben, und unter allen Vorstellungen die Verbindung die einzige ist, die nicht durch Objekte gegeben,

25 Ebd. 
sondern nur vom Subjekte selbst verrichtet werden kann“. ${ }^{26}$

Wenn Kant von der „Verbindung“ eines Mannigfaltigen spricht, dann hat er folgende Art der Verbindung von mehreren Vorstellungen im Sinn: Indem wir sie verbinden, stellen wir etwas als „[in demjenigen] Objekt verbunden“ vor, zu dem diese Vorstellungen gehören.

Überlegen wir zuerst, warum eine derartige Verbindung nicht durch die Sinne in uns kommen kann. ${ }^{27}$ Nehmen wir an, die relevante Verbindung von Vorstellungen ist folgende:

(A) Dieser Stein dort drüben ist schwer,

wobei die gegebene Erkenntnis, die an der Subjektposition vorgestellt wird, von einem großen Stein handelt, der dort drüben liegt, und die mit einer Vorstellung von Schwere so verknüpft werden soll, dass der Stein als schwer vorgestellt wird. Dafür ist es nun offensichtlich nicht hinreichend, dass ich einerseits den Stein, andererseits Schwere vorstelle: Ich könnte das tun, ohne irgendeinen Gedanken über ihre Verbindung miteinander zu denken. Auch scheint es nicht hinreichend, dass meine Vorstellung des Steins und meine Vorstellung von Schwere in irgendeiner besonderen Beziehung zueinander in meinem eigenen Bewusstseinsstrom stehen (z.B. Gleichzeitigkeit, Ursache-undWirkung, etc.). Es ist nicht erforderlich, dass zwei unterschiedliche Vorstellungen in einer psychologischen Relation stehen, sondern eine einzelne, einheitliche Vorstellung davon, dass dieser Stein schwer ist. Eine Assoziation von Vorstellungen ist aber keine Vorstellung von Assoziation. Wenn ich den Stein als schwer vorstellen soll, dann muss ich den Stein und Schwere, wie Kant sagt, „,in einem Bewusstsein“ vorstellen: d.h. in einer einzelnen Ausübung meiner Vorstellungskraft. In dieser einzelnen, einheitlichen Vorstellung muss es aber trotzdem möglich sein, die Elemente zu unterscheiden, die auch getrennt auftreten oder in einem Urteil verknüpft sein können, das unverbindlicher ist, wie z.B.:

(B) Als ich diesen Stein dort drüben hochgehoben habe, hat er sich schwer für mich angefühlt. ${ }^{28}$ In (B) wird Schwere mit dem Stein nicht als ,im Objekt verbunden“ vorgestellt. Ich kann mich gerade in einem erschöpften Zustand befinden, sodass sich selbst eine leichte Belastung schwer für mich anfühlen würde. (B) lässt diese Möglichkeit zu: Es verbindet dieselben Vorstellungselemente,

$26 \mathrm{KrV}, \mathrm{B} 129-130$.

27 Die folgende Diskussion sollte mit Kants Bemerkungen bei B130-131 und B141-142 verglichen werden.

28 Der Einfachheit halber betrachte ich ein Urteil, das eine besondere Gelegenheit betrifft, in der man einen Stein hebt. Kants eigenes Beispiel lautet wie folgt: „Wenn ich einen Körper trage, fühle ich einen Druck der Schwere“ (KrV, B142). Es liegt nahe, dies so aufzufassen, dass hier eine konstante subjektive Assoziation zwischen Begegnungen mit einer bestimmten Art von Sache, Körpern und einem bestimmten Eindruck, Schwere, behauptet wird. Kants Punkt ist, dass selbst ein Urteil, das solch eine gesetzesartige Assoziation von Eindrücken behauptet, nicht mit dem Urteil äquivalent ist, dass Körper schwer sind. 
die auch in (A) auftauchen, aber auf eine andere, unverbindlichere Weise. So wie es aussieht, wird durch das, was meine Sinne mir präsentieren - den sicht- und fühlbaren Stein, die fühlbare Erfahrung des Gewichts -, nicht bestimmt, wie ich sie verbinden muss. Oder um es in einer noch kantischeren Sprache auszudrücken: sie bestimmen nicht, welchen „Begriff der Verbindung“ ich anwenden muss. Im Fall von (A) würde Kant sagen, dass ich den Stein unter die Kategorie der Substanz und Schwere unter die Kategorie des Akzidenz bringe und sie somit in einem Urteil verbinde, das Anspruch auf objektive Gültigkeit erhebt. In (B) bringe ich sie nicht auf diese Weise unter Kategorien. Welcher unserer Sinne könnte uns mit den Begriffen der Verbindung ausstatten, die hier den Unterschied ausmachen? Kants Antwort lautet: keiner. Das soll aus der Idee sinnlicher Rezeptivität folgen: Sie ist Quelle einer Mannigfaltigkeit von Vorstellungen, die durch die Zufälligkeiten meiner einzelnen Begegnungen mit Gegenständen bedingt sind. Um von solch einer Mannigfaltigkeit zu einer einheitlichen Vorstellung überzugehen, was der Fall ist, braucht es einen Beitrag von einem nicht-rezeptiven Vermögen, dem Verstand.

Die Aktivität, durch die der Verstand einer Mannigfaltigkeit von Vorstellungen Einheit verleiht, nennt Kant „Synthesis“ und er behauptet von dieser synthetisierenden Aktivität: ,[S]eine [des Verstandes] Synthesis [ist], wenn er für sich allein betrachtet wird, nichts anders, als die Einheit der Handlung, deren er sich, als einer solchen, auch ohne Sinnlichkeit bewusst ist““ ${ }^{29}$ Kants Behauptung, dass sich der Verstand der Einheit seines Akts ,als einer solchen“ bewusst ist, scheint Folgendes zu beinhalten: Der Verstand ist sich der Einheiten, die er hervorbringt, ,,auch ohne Sinnlichkeit“" bewusst, weil er den verschiedenen Vorstellungen selbst aktiv Einheit verleiht. In einer Hinsicht verstehe ich dies so, dass es Allisons Interpretation widerspricht. Wie wir gesehen haben, versucht Allison, den Sinn, in dem Urteile aktiv sind, zu erklären, indem er sich auf die Idee beruft, dass zu urteilen notwendig Gegenstand der Apperzeption ist; in der eben zitierten Passage scheint Kant aber die umgekehrte Reihenfolge der Erklärung nahezulegen: nämlich, dass wir uns genau deshalb unserer eigenen Akte der Synthesis apperzeptiv bewusst sind, weil sie unsere eigenen Akte sind. Ich denke, dass man Kants wirkliche Einsicht in den Fokus rückt, wenn man in dieser Hinsicht von Allisons Lesart abweicht.

Unser Problem bestand darin, dass es uns an einem klaren Verständnis der Natur unseres apperzeptiven Bewusstseins und seiner Verknüpfung mit dem aktiven Charakter unserer Erkenntnis fehlte. Der Vorschlag, den wir jetzt prüfen, lautet: Das relevante Bewusstsein ist eines, das wir genau dadurch haben, dass wir die aktive Quelle der relevanten Akte der Synthesis sind. Konzentrieren wir uns auf den Fall der Synthesis von Vorstellungen im Urteil, dann ist die Idee also

$29 \mathrm{KrV}, \mathrm{B} 153$. 
nicht bloß, dass wir $S$ ist $P$ nur urteilen, wenn wir uns bewusst sind, $\mathrm{S}$ als $\mathrm{P}$ aufzufassen (Allisons Apperzeptionsbedingung); die Idee ist, dass unser Bewusstsein davon, dass wir $S$ ist $P$ urteilen, die besondere Art von Bewusstsein ist, über die jemand verfügt, der tätig ist, indem er diese Vorstellungen bewusst verbindet. Um den Gehalt dieser Idee zu begreifen, ist es hilfreich, unser Bewusstsein von unseren eigenen Urteilen mit unserem Bewusstsein von z.B. unseren eigenen Schmerzen zu kontrastieren. Es ist plausibel, dass bei selbstbewussten Wesen eine Variante von (AB) nicht nur für Urteile, sondern auch für Schmerzen gilt: Wir fühlen Schmerz nur dann, wenn wir uns bewusst sind, dass wir Schmerzen fühlen. Es besteht kein Zweifel, dass einige Philosophen diese Behauptung über Schmerzen bestreiten würden. Für meine gegenwärtigen Zwecke ist aber entscheidend, dass selbst Philosophen, die sie akzeptieren, hieraus wohl nicht folgern würden, dass Schmerz eine Ausübung unserer kognitiven Tätigkeit ist. Aus dem Umstand, dass eine bestimmte Art von mentalem Ereignis notwendig Gegenstand der Apperzeption ist, folgt nicht, dass es sich um einen Akt handelt, der in irgendeinem interessanten Sinn spontan ist. Sich eines mentalen Ereignisses als eines selbstvollzogenen Akts bewusst zu sein, verlangt etwas Zusätzliches: nicht bloß die unmittelbare Apperzeption seiner Existenz, sondern ein in spezifischer Weise tätiges [agential] Bewusstsein der eigenen Rolle in der Bestimmung seines Seins [in determining it to be]. Unsere Diskussion des Unterschieds zwischen (A) und (B) hilft zu verdeutlichen, um welche Art von Bewusstsein es sich handeln könnte. Kant betrachtet den Unterschied zwischen dem Urteil, dass der Stein sich lediglich für mich schwer anfühlt, und dem Urteil, dass der Stein selbst schwer ist, als einen Unterschied in Hinsicht auf die Weise, in der ich die Verbindung meiner Vorstellung des Steins und meiner Vorstellung von Schwere als gültig betrachte. ${ }^{30}$ Im Urteil, dass der Stein sich für mich schwer anfühlt, stelle ich die Verbindung dieser Vorstellungen als bloß „subjektiv gültig“ vor: als etwas, das bloß in Hinsicht auf meinen eigenen Bewusstseinszustand erforderlich ist. Im Urteil, dass der Stein selbst schwer ist, stelle ich die Verbindung als „objektiv gültig“ vor: als etwas, das in jedem fehlerfreien Urteil über das Gewicht dieses Steins erforderlich ist. Kant meint, dass mein Urteil Der Stein ist schwer genau diesen Gedanken zum Ausdruck bringt: dass diese Verbindung der Vorstellung dieses Steins und der Vorstellung von Schwere in jedem Urteil über diese Angelegenheit erforderlich ist. Genau hierin besteht, wie er sagt, die Aufgabe der Kopula „,ist“: „die objektive Einheit gegebener Vorstellungen von der subjektiven zu unterscheiden““. ${ }^{31}$ Die Kategorien der Substanz und des Akzidenz - auf der einen Seite der Begriff von etwas, das in der Anschauung präsentiert wird und immer als Subjekt, niemals bloß als Prädikat aufzufassen ist, und

$30 \mathrm{Vgl} . \mathrm{KrV}, \mathrm{B} 142$.

31 Vgl. KrV, B141-142. 
auf der anderen Seite der Begriff von etwas, das als Prädikat eines grundlegenderen Subjekts aufzufassen ist - sind die Standards, die diesen Akt der Synthesis leiten: sie artikulieren das Maß, das das Subjekt anzuwenden sucht, wenn es die relevante Mannigfaltigkeit von Vorstellungen ordnet.

Betrachten wir nun den Charakter des Bewusstseins eines Subjekts, das ein derartiges Urteil fällt. Gemäß der gerade erfolgten Erklärung bringt sein Urteil

(A) Dieser Stein dort drüben ist schwer

zum Ausdruck, dass es diese Verbindung von Vorstellungen als etwas betrachtet, das in jedem gültigen Urteil über diese Angelegenheit erforderlich ist, und zwar aufgrund der Standards, die in den Kategorien der Substanz und des Akzidenz enthalten sind. Und genau weil das Subjekt denkt, dass es von jedem gefordert ist, so über diese Angelegenheit zu urteilen, urteilt es selbst: der Umstand, dass es diese Vorstellungen als so zu verbinden auffasst, ist identisch damit, dass es sie so verbindet. Ich schlage vor, dass es dieser Punkt ist, der unserer Intuition zugrunde liegt, wonach zu urteilen eine Form des selbstbewussten „Auffassens von etwas als etwas“ ist. Der Punkt ist nicht bloß, dass das Urteil $S$ ist $P$ notwendig das Bewusstsein enthält, dass man $S$ ist $P$ urteilt; der Punkt ist, dass im Urteil $S$ ist $P$ notwendig enthalten ist, dass man eine spezifische Verbindung von $S$ ist $P$ als erforderlich begreift und sich dadurch bewusst ist, dass man das eigene Urteil bestimmt. Ein Subjekt ist sich seines eigenen Urteils also in einer spezifischen Weise bewusst: nicht im Sinne einer gegebenen Tatsache, sondern im Sinne von etwas, dessen Sosein es selbst bestimmt. In diesem Sinn ist das Bewusstsein des Subjekts von seinem Urteil auf spezifische Weise tätig.

All dies verdient eine viel ausführlichere Diskussion, als ich sie hier leisten kann. Ich möchte bloß anmerken, inwiefern diese Beobachtungen helfen, die Schwierigkeiten aufzulösen, mit denen Allisons Lesart konfrontiert ist. Ein Problem bestand darin, dass wir kein befriedigendes Argument von $(\mathrm{AB}) \mathrm{zu}(\mathrm{ST})$ finden konnten. Unsere überarbeitete Theorie vermeidet diese Schwierigkeit, weil sie nicht versucht, für (ST) auf Grundlage von (AB) zu argumentieren. Sie behauptet vielmehr, dass wir genau deshalb ein (spezifisch tätiges) Bewusstsein unserer eigenen Urteile haben, weil diese unsere eigenen Akte sind. Der aktive Charakter von Urteilen erscheint also nicht als eine Konsequenz davon, dass Urteile Gegenstand der Apperzeption sind, sondern vielmehr als Grund der spezifischen Form ihrer Apperzeption.

Ein weiteres Problem bestand darin, dass es sich als schwierig erwiesen hat, eine Lesart von $(\mathrm{AB}) \mathrm{zu}$ identifizieren, die gleichzeitig plausibel und klar ist. Gemäß unserer überarbeiteten Erläuterung besteht das Urteil $S$ ist $P$ darin, dass man eine bestimmte Verbindung der Vorstellungen $\mathrm{S}$ und $\mathrm{P}$ in bewusster Weise als erforderlich auffasst. Da diese Verbindung von Vorstellungen als 
erforderlich aufzufassen dasselbe ist, wie $S$ ist $P$ zu urteilen, gilt Folgendes: Ein Subjekt, das die Verbindung $S$ ist $P$ in einer bewussten Weise als erforderlich begreift und ferner versteht, was es heißt zu urteilen, befindet sich in der Lage zu wissen, dass es selbst $S$ ist $P$ urteilt. Gleichzeitig vermeiden wir das unplausible Bild, wonach die gewöhnliche Orientierung desjenigen Bewusstseins, das in seinem Urteil enthalten ist, sich nach Innen, auf seine eigene kognitive Aktivität richtet. Im gewöhnlichen, unreflektierten Fall liegt der Fokus des Subjekts nicht auf dem, was es selbst tut, sondern darauf, welche Verbindung einer bestimmten Mannigfaltigkeit von objektiv-gehaltvollen Vorstellungen korrekt ist. Indem es hierüber eine Bestimmung trifft, urteilt das Subjekt und kann, wenn es darüber nachdenkt, ,,sein Urteil durch das Ich denke begleiten“. Sein Bewusstsein ist aber nicht in erster Linie ein betrachtendes Bewusstsein von der Existenz eines bestimmten Vorstellungsakts, sondern ein tätiges Bewusstsein davon, dass es eine bestimmte Frage klärt.

Abschließend gilt es zu bemerken, dass nichts von dem, was wir gesagt haben, uns darauf verpflichtet, ein Urteil als ein Ereignis zu betrachten, das eine Überzeugung hervorbringt. Die Art von tätigem Bewusstsein, die wir beschrieben haben, ist eine, die ein vernünftiges Subjekt immer dann ausübt, wenn es etwas für wahr hält. Demnach können wir sagen, dass seine kognitive Tätigkeit nicht nur in der Entstehung mancher seiner Überzeugungen am Werk ist, sondern im generellen Charakter seines Überzeugtseins. Als Subjekt, das über das Vermögen des Verstandes verfügt, ist es fortwährend damit beschäftigt herauszufinden, inwiefern ihm die Mannigfaltigkeit von Vorstellungen, die es durch seine Sinne erhält, Aspekte einer verständlichen Welt präsentiert. Dies herauszufinden erfolgt in großen Teilen ohne reflektierendes Nachdenken. Und trotzdem ist hierin enthalten, dass das Subjekt bestimmte Haltungen darüber einnimmt, wie die Dinge zusammenpassen. Mit diesen Haltungen geht einher, dass das Subjekt aus verschiedenen möglichen Formen der Verbindung in der Weise auswählt, die durch den Kontrast zwischen (A) und (B) illustriert wurde. Dabei geht es nicht bloß um einen Akt, durch den bejaht wird, dass etwas so ist, sondern darum, dass in fortdauernder Weise, eine umfassende, zusammenhängende und sich im Wachsen befindende Sicht darüber aufrecht erhalten wird [holding], wie die Dinge stehen. Dieses Aufrechterhalten ist, wie ich glaube, der primäre Ort kognitiver Aktivität bei Kant. ${ }^{32}$

Aus dem Englischen von Jonas Zahn.

\section{Literatur}

32 Ich verdanke die Rede vom „Aufrechterhalten“ [holding] Engstrom (2009), 103-104. 
Allison, H. (1996a), Kant's Refutation of Idealism, in: ders., Idealism and Freedom, New York.

Allison, H. (1996b), Naturalizing Kant's Transcendental Psychology, in: ders., Idealism and Freedom, New York.

Boyle, M. (2009), Two Kinds of Self-Knowledge, in: Philosophy and Phenomenological Research $58(1), 133-164$.

Boyle, M. (2011a), Active Belief, in: Canadian Journal of Philosophy, Supplementary Vol. 35, 119148.

Boyle, M. (2011b), Making up Your Mind and the Activity of Reason, in: Philosopher's Imprint 11 (17), 1-24.

Engstrom, S. (2006), Understanding and Sensibility, in: Inquiry 49 (1), 2-25.

Engstrom, S. (2009), The Form of Practical Knowledge, Cambridge.

Frege, G. (1919), Die Verneinung, in: ders., Logische Untersuchungen (hrsg. von Günther Patzig), Göttingen 2003, 63-83.

Kant, Immanuel (1781/1787), Kritik der reinen Vernunft, Hamburg 1998.

Kimhi, I. (im Erscheinen), Thinking and Being, Cambridge.

Longuenesse, B. (1998), Kant and the Capacity to Judge, Princeton.

McDowell, J. (1994), Mind and World, Cambridge.

McDowell, J. (1998), Having the World in View: Sellars, Kant, and Intentionality, in: Journal of Philosophy 95 (9), 431-492.

Shoemaker, S. (2009), Self-Intimation and Second Order Belief, in: Erkenntnis 71, 35-51.

Thomas von Aquin, Summa Theologica, übers. von Dominikanern und Benediktinern, vollständige ungekürzte Ausgabe, Salzburg 1933. 